Please do not remove this page

RMIT

UNIVERSITY

\title{
A mobile phone based intelligent telemonitoring platform
}

Sufi, Fahim; Fang, John; Mahmoud, Seedahmed; Cosic, Irena

https://researchrepository.rmit.edu.au/esploro/outputs/9921860029601341/filesAndLinks?institution=61RMIT_INST\&index=null

Sufi, F., Fang, J., Mahmoud, S., \& Cosic, I. (2006). A mobile phone based intelligent telemonitoring platform. Proceedings of the 3rd IEEE-EMBS International Summer School and Symposium on Medical Devices and Biosensors. https://doi.org/10.1109/ISSMDBS.2006.360108

Published Version: https://doi.org/10.1109/ISSMDBS.2006.360108

Repository homepage: https://researchrepository.rmit.edu.au

(c) 2006 IEEE. Personal use of this material is permitted. However, permission to reprint/republish this material for advertising or promotional purposes or for creating new collective works for resale or redistribution to servers or lists, or to reuse any copyrighted component of this work in other works must be obtained from the IEEE.

Downloaded On 2023/04/26 11:13:59 +1000 


\title{
A Mobile Phone Based Intelligent Telemonitoring Platform
}

\author{
Fahim Sufi, Qiang Fang, Seedahmed S. Mahmoud and Irena Cosic
}

\begin{abstract}
In this paper, we propose a generic smart telemonitoring platform in which the computation power of the mobile phone is highly utilized. In this approach, compression of ECG is done in real-time by the mobile phone for the very first time. The fast and effective compression scheme, designed for the proposed telemonitoring system, outperforms most of the real-time lossless ECG compression algorithms. This mobile phone based computation platform is a promising solution for privacy issues in telemonitoring through encryptions. Moreover, the mobile phones used in this platform performs preliminary detection of abnormal biosignal in realtime. Apart from the usage of mobile phones, this platform supports background biosignal abnormality surveillances using data mining agent.
\end{abstract}

Keywords-Telemonitoring, J2ME, Mobile Phone Computing, ECG Compression

\section{INTRODUCTION}

$\mathrm{M}$ ORE than half of the 8000 home care agencies in US are currently using some forms of telemonitoring [1]. Only in US, sales of services and devices associated with telemonitoring are projected to rise from $\$ 461$ million in 2005 to over $\$ 2.5$ billion in 2010 [1]. Since monitoring at hospital premises is financially challenging than home monitoring, more people are getting interested in receiving telemonitoring services. Moreover, telemonitoring gives them freedom of staying home and living normal life with their families. Hospitals with limited healthcare resources also benefits from telemonitoring service because it can accommodate more serious conditioned patients who need emergency attention.

Coronary heart disease (CHD, or ischaemic heart disease) is the most common cause of sudden death in Australia [2]. Its main manifestations consist of Acute Myocardial Infarction (AMI, or heart attack) and angina. Stroke (or cerebrovascular disease) is Australia's second greatest killer after CHD and the leading cause of long term disability in adults [2]. Heart failure is a major burden on society due to its high costs of care, lower quality of life and premature death (the third biggest CVD killer). Among Australians having a heart attack, about $25 \%$ die within an hour of their first-ever symptoms and over $40 \%$ will be dead within a year [2]. So, for the $40 \%$ who would generally be dead in one year, telemonitoring can be a life saver.

Management of chronic diseases, such as diabetes, costs a

F. Sufi, Q. Fang, S. Mahmoud and I. Cosic are with the School of Electrical \& Computer Engineering, RMIT University, Melbourne, VIC 3001, Australia (Q. Fang, phone: +61 3 99252432; fax: +61 3 99252007; email: john.fang@rmit.edu.au). lot of money. Sometimes these diseases are associated with other disorders, for which Point of Care Testing (POCT) are needed, so that, the effect of other disorders can be controlled [3]. Several telemonitoring systems have been proposed for monitoring various patient groups, e.g., chronic patient [4]-[13], acute patient [7]-[13], disaster affected patient [14] and elderly person [15]-[16]. Moreover, the necessity of telemonitoring for performance measurement of special groups, like military personnel, is also crucial [17]. Recently, various rescuer monitoring systems have been designed for military, athlete, public transport driver, fire fighter, police, and adventurer [8], [15] and [18].

In this paper, a unique telemonitoring platform is proposed, which is generic and can be easily adopted in different telemonitoring scenarios. The proposed platform uses the processing power of mobile phone carried by both users, e.g., Chronic Patient, and the service providers, e.g., the medical doctors. Java ${ }^{\mathrm{TM}}$ based software running on the mobile phones performs computation intensive tasks like biosignal analysis, compression, encryption and detection of abnormal pattern. The proposed system automatically alerts medical service provider, when medical assistance is crucial for the user.

\section{ARCHITECTURE DESIGN}

The architecture of our system is illustrated in Fig. 1. The core communication is done through the mobiles phones carried by both user and telemonitoring service provider. Most of the mobile phones now support compact Java ${ }^{\mathrm{TM}}$ runtime called Kilobyte Virtual Machine (KVM). KVM is a miniature run time that can run Java ${ }^{\mathrm{TM}}$ software called MIDlets in the mobile phone.

Current mobile phones possess considerable computation powers which can perform computations for 3D games, MP3 and MPEG encoding and decoding. Even, Optical Character Recognition software is tested on current mobile phones [19]. The proposed platform makes use of this processing power for the use of telemonitoring. The proposed system contains five main sub systems. These sub systems are: patient's unit, doctor's unit, web server, database and web mining agent.

\section{A. Patient's Unit}

This unit contains biosignal acquisition device and mobile phone. Our own developed biosignal acquisition device with ECG, Oximetry, skin impedance and blood pressure sensors connects to the MIDlet software running on the patient's mobile via Bluetooth streaming. For the Bluetooth 


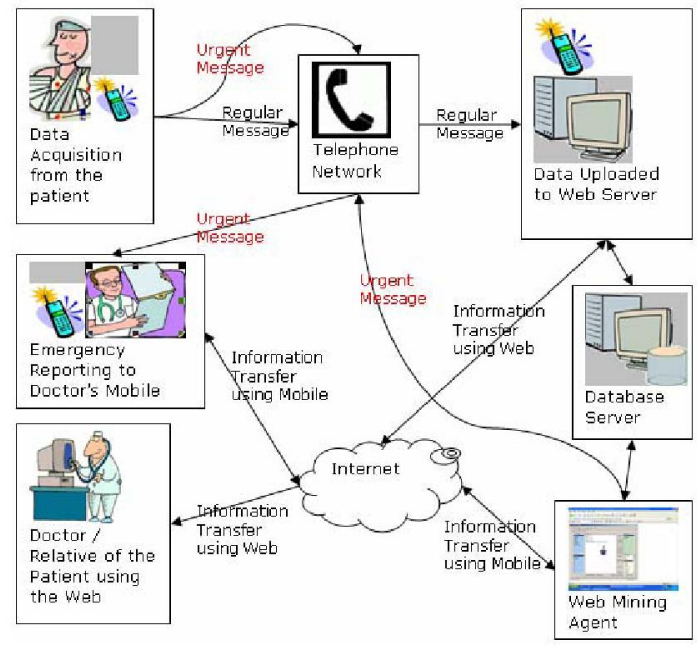

Fig. 1. Architecture of the proposed system

connectivity, JSR-82 [20] specification was used.

Recently, a very new interconnection technology, Near Field Communication (NFC) is being researched and on its way to be commercialised [21]. Study shows that the new generation NFC-Enabled phone will be widely available within next four years [22]. Like Bluetooth, NFC based communication can easily be implemented to the proposed system, since the support for NFC with J2ME is already in progress through JSR-257 [20].

Third party sensors can also be supported for serving specialized purposes. Even, embedded mobile phone sensors can be efficiently used through JSR-256 [20].

\section{B. Doctor's Unit}

This unit is the specialised MIDlet software, running on doctor's mobile. It serves the medical service provider's request of patient information. It notifies the doctor in case of an emergency situation, when medical help is required the most. Custom reporting, charting and analysis of patient's condition for the selected period can be performed by the doctor's unit.

\section{Web Server}

This unit provides restricted information to the authorised medical personnel or even relatives in case of elderly monitoring.

\section{Database}

All the biological signals from the patent's unit are stored here, efficiently. For implementation purposes we have chosen SQL Server 2000, because of its support of data mining techniques (like, Clustering, Decision tree etc.) with Online Analytical Processing (OLAP) module.

\section{E. Web Mining Agent}

This agent is responsible for providing requested information to the doctor's mobile. Since, our system is designed as a generic system it supports all the phones supporting Java ${ }^{\mathrm{TM}}$. Again, each phone (doctor's unit) is different in their display size, supported colours or even some operations. Web mining agent automatically analyses these information and format them to be suitable for the doctor's mobile screen.

Moreover, web mining agent performs background data mining operations to analyse the stored information of patient. If it discovers some pattern demanding urgent diagnosis, it can inform the doctor via SMS.

\section{SYSTEM FUnCTIONS}

The proposed system supports several functions, since it is designed as a generic platform, supporting multiple applications. Fig. 2 and Fig. 3 show the screen shots of the patient's phone and the doctor's phone respectively.

\section{A. Regular Signal Submission}

During this normal operation mode, patient's unit sends the biological signal acquired from the acquisition device at a regular interval to the Web Server. During this signal acquisition and submission process the intelligent wireless software running on patient's mobile performs analysis of any abnormal pattern.

\section{B. Notifying Emergency Situation}

This situation is triggered when the software on patient's mobile detects any abnormality on the signal or the web mining agent discovers any pattern requiring urgent medical attention. Depending on the urgency of the situation, the system is capable of notifying the appropriate medical personnel for immediate attention via SMS.
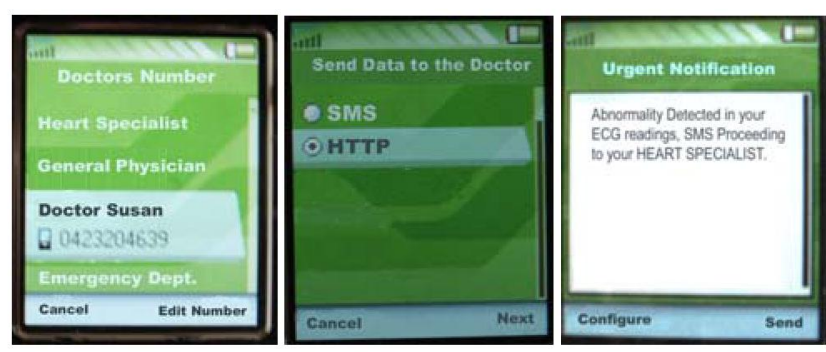

Fig. 2. Screen shots of the User Interface of patient's mobile phone

\section{Real-Time Monitoring}

During some emergency situations, if the doctor wants to initiate a real-time monitoring to provide special advice to the remote patient, he can do so by this mode of operation. Instead of submitting the vital signals at a regular interval, the patient's mobile starts to send the collected data in streaming mode.

\section{Detection Algorithm}

The MIDlet software running in the patient's mobile phone has a rule based system, which can distinguish abnormal situations like excessive pulse rate, longer $R R$ interval at the ECG signal etc. Patient's mobile phone performs real-time detection. 
Moreover, the web mining agent performs powerful data mining applications in background. Since, the web mining agent works on the stored data of the patient, it can assess whether the patient's condition is degrading drastically over time.

\section{E. Compression algorithm}

The proposed system uses its unique lossless compression scheme, which can provide $70 \sim 75 \%$ compression rate, which is substantially higher than some existing lossless real-time compression scheme [7]. The 50 60\% compression ratio of RECAD, was performed in PC / PDA. But, our proposed mobile phone based solution out performed RECAD by much more efficient compression scheme. Since, this platform supports background detection by web mining agent, lossless data is needed for accurate detection. The compression algorithm removes the floating point information, since MIDlet applications are unable to handle floating point operations. Then it performs some simple mathematical operations, followed by a symbol substitution based compression suitable for generic ECG signals.

\section{F. Efficient Retrieval of Information}

The software running on doctor's mobile is capable to support multiple report generation. It lets the doctor to select the name of the patient, the information to be displayed (like, the blood pressure, pulse, breathing component etc.), the mode of display, etc. Graphs are dynamically plotted on the mobile screen. So, if the doctor wants to zoom in a particular portion of the graph or even perform amplification or shifting, he can do so by using the gaming keys in his mobile phone.

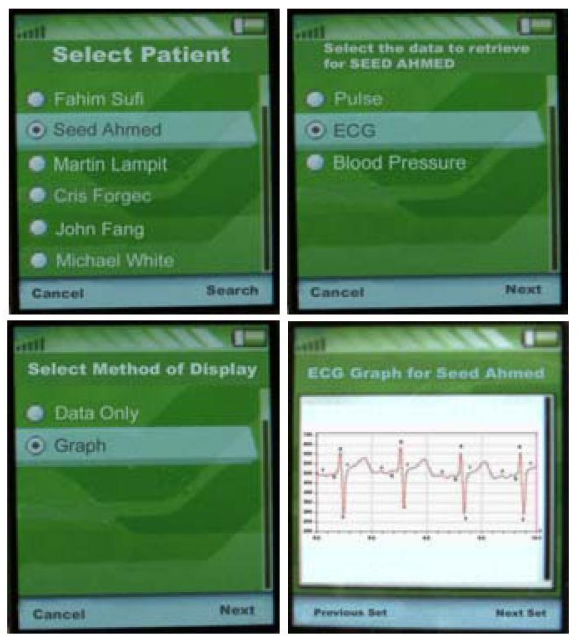

Fig. 3. Screen shots of the User Interface of doctor's mobile phone

Some WAP based implementations [13], where the ECG signals were sent as a picture message to the doctor's mobile, suffer serious limitations like, usage of huge bandwidth due to the big file size, inability to zoom particular image or particular section etc. Those [13] implementation used doctor's mobile for taking only inputs from the doctor, and all the processing were done on the server end. So, it introduces huge usage of network bandwidth that leads to network congestion. Whereas, the proposed system doesn't need to supply request to the server multiple times, it merges all the required information, encode them for network bandwidth efficiency and send it to the server.

In addition, the web server enables viewing of permitted information to the relatives or medical personnel using browsers on a PC.

\section{G. Authentication \& Encryption}

Usage of J2ME unleashes the possibility of PC based encryption techniques like symmetric, asymmetric (Public Key, Private Key based), digital signature, digital time stamping available for the purpose of health monitoring scenario. The urgent requirement of encryption for health related information has been well researched elsewhere [15]. With the advent of the proposed platform, encryptions of biosignals can be performed with third party J2ME cryptography toolkits like, Bounty Castle Lightweight API, IAIK ME JCE, Phaos Technology Micro Foundation Toolkit, NTRU jNeo for Java Toolkit, B3 digital signature scheme etc. [23].

Securing the acquisition device to patient's mobile communication was done via detection of 6-byte long globally unique Bluetooth Device Address (BDA), authentication authorization, encryption and PIN exchange. But, in urgent scenario, this long authorization process can be simplified using the RFID based touch scheme provided by NFC [21]. Simply by touching the NFC supported mobile with the acquisition device, a Bluetooth streaming link between them can be initiated within seconds.

\section{DISCUSSION AND CONCLUSION}

In this paper we propose a generic mobile phone based intelligent telemonitoring platform with good extensibility. The proposed platform makes it easy to change the functionalities or telemonitoring applications, just by updating the program by Over-the-Air (OTA) deployment [23] of MIDlets to the mobile. So, the system can be adopted for different applications with minimal effort. Most of all, J2ME based system reveals standard and different third party APIs for performing complex computations like, compression, encryption, streaming multimedia for the service of telehealth of the mobile phone. The proposed platform provides flexibility in terms of wireless communication, from the biological signal acquisition device to mobile through Bluetooth and from the patient to service provider communication via $2.5 \mathrm{G}$ or $3 \mathrm{G}$ network.

The proposed system enables the doctor to receive and analyse the patient report and also, deliver doctor's treatment and specialist advice to remote patient. The doctor doesn't need to be sitting in front of a stationary computer 
within the medical facility. The proposed system uses smart client technology [23] instead of thin client technology used by some [13] WAP based system. So, it can use the network bandwidth much more efficiently. Moreover, it doesn't need any physical wired internet connection like some systems [4], [10]-[11], [13], [16] and [18], making the proposed system a true wireless solution. The sending of physical parameters from the acquisition device is automated by the MIDlet running in patient's mobile, making the whole system less error prone compared to some existing systems [7]. Finally the proposed system is based on generic mobile phone (costing $100 \mathrm{~s}$ of dollars) supporting $\mathrm{Java}^{\mathrm{TM}} \mathrm{KVM}$, instead of expensive Pocket PC or Smart phone (1000s of dollars) based solution. Therefore, cost effectiveness is another crucial advantage that the proposed system provides.

The generic telemonitoring platform presented here is a cost effective, flexible and robust solution supporting a unique mobile based computational platform where compression, detection and encryption are some of the possibilities.

\section{ACKNOWLEDGEMENT}

This research was supported by an Australian Postgraduate Award and a Victorian Govt. Information \& Communication Technology Award.

\section{REFERENCES}

[1] R. Roberts. Use of Remote Monitoring Devices Increases. Telemedicine Information Exchange (Original Source: Wall Street Journal, April 18, 2006). [Online]. Available: http://tie.telemed.org/legal/news.asp

[2] Access Economics Pty Limited, The shifting burden of cardiovascular disease in Australia, A report of Heart foundation, 2005 [Online]. Available: www heartfoundation.com.au media/nhfa_shifting_burden_cvd_0505.pdf

[3] C. A. Lehmann, "The future of home testing- implications for traditional laboratories", Clinica Chimica Acta, 323(1-2), 2002, pp 2136

[4] Y. Y Chen, Y. T Wu, C. C Wu, S. M Wu, F. S Jaw, Development of wireless blood glucose meter and diabetes self-management system, Proceedings of the 26th Annual International Conference of the IEEE EMBS, pp. 3384-3386, September 2004

[5] O. Lynch, J. McGrory and E. Coyle, "Design of Mobile phone application for point of care test result validation", Proceeding of the LASTED International Conference of Telehealth, July 2005

[6] E. Jovanov, A. Lords, D. Raskovic, P. Cox, R. Adhami, F. Andrasik, "Stress Monitoring Using a Distributed Wireless Intelligent Sensor System," IEEE Engineering in Medicine and Biology Magazine, Vol. 22, No. 3, pp. 49-55, May/June 2003

[7] H. Zhou, K. M. Hou, J. Ponsonnaille, L. Gineste and C. D. Vaulx, "A Real-Time Continuous Cardiac Arrhythmias Detection System: RECAD", Proceedings of the 27th Annual Conference of IEEE Engineering in Medicine and Biology, September 2005

[8] Y. Jasemian and L. Arendt-Nielsen, "Evaluation of a realtime, remote monitoring telemedicine system using the Bluetooth protocol and a mobile phone network", Journal of Telemedicine and Telecare, Vol. 11, No. 5, pp. 256-160, 2005

[9] E Zhao and L. Cui, "EasiMed: A remote health care solution", Proceedings of the 27th Annual Conference of IEEE Engineering in Medicine and Biology, September 2005

[10] W. Jin-gang, S. Xiao-Bo1, W. Ping, H. Wei and D. Cui-Lian, "Remote Heart Sound Monitoring System", Proceedings of the 27th Annual
Conference of IEEE Engineering in Medicine and Biology, September 2005

[11] M. Braecklein, I. Tchoudovski, C. Moor, K. Egorouchkina, L.P. Pang and A. Bolz, "Wireless Telecardiological Monitoring System for the Homecare Area", Proceedings of the 27th Annual Conference of IEEE Engineering in Medicine and Biology, September 2005

[12] W. Chen, D. Wei, M. Cohen, S. Ding, S. Tokinoya, and N. Takeda, "Development of a Scalable Healthcare Monitoring Platform", Proceedings of the Fourth International Conference on Computer and Information Technology (CIT'04), 2004

[13] K. Hung and Y. T. Zhang, "Implementation of a WAP-based telemedicine system for patient monitoring", IEEE Transactions on Information Technology in Biomedicine, Vol. 7, No. 2, , pp. 101- 107, June 2003

[14] T. Gao, D. Greenspan, M. Welsh, R. R. Juang and A. Alm, "Vital Signs Monitoring and Patient Tracking Over a Wireless Network", Proceedings of the 27th Annual Conference of IEEE Engineering in Medicine and Biology, September 2005

[15] K. J. Patel, "Development of a new healthcare monitoring system for elderly using emerging communication technologies", Proceedings of the IASTED International Conference on Telehealth, pp 50-54, July 2005

[16] J. S. Shieh, C. T. Chuang, X. Wang, P. Y. Kuo, "Web-Based Remote Monitoring Health of the elderly via mobility changes using frequency and rank order statistics", Proceeding of the LASTED International Conference of Telehealth, July 2005

[17] Sgt. 1st Class Doug SampleAmerican Forces Press Service (14 Feb, 2003). Defense alters deployment health monitoring. [Online] Available:

http://www.demilitary.com/army/pentagram/8_06/health/21612-1.html

[18] C. Glaros, D.I. Fotiadis, A. Likas and A. Stafylopatis, "A wearable intelligent system for monitoring health condition and rehabilitation of running athletes", Proc. 4th IEEE Conf. on Information Technology Applications in Biomedicine, pp. 276-279, 2003

[19] D. Graham-Rowe (14 September, 2005). Camera phones will be highprecision scanners. NewScientist.com news service. [Online]. Available: http://www.newscientist.com/article.ns?id=dn7998

[20] JSRs: Java Service Requests. Java Community Press Web Site. [Online]. Available: http://www.jcp.org/en/jst/overview

[21] NFC Forum web site. http://www.nfc-forum.org/

[22] Near Field Communications (NFC). Simplifying and Expanding Contactless Commerce, Connectivity, and Content. ABI Research, Oyster Bay, NY, 4Q2005

[23] M. J. Yuan, Enterprise J2ME: developing mobile Java, Upper Saddle River, NJ: Prentice Hall PTR, c2004. 